\title{
Mapping a major QTL responsible for dwarf architecture in Brassica napus using a single-nucleotide polymorphism marker approach
}

Yankun Wang ${ }^{1,2+}$, Wenjing Chen ${ }^{1,2+}, \mathrm{Pu}_{\mathrm{Ch}}{ }^{1,2+}$, Shubei Wan ${ }^{1,2}$, Mao Yang ${ }^{1,2}$, Mingming Wang ${ }^{1,2}$ and Rongzhan Guan ${ }^{1,2^{*}}$

\begin{abstract}
Background: Key genes related to plant type traits have played very important roles in the "green revolution" by increasing lodging resistance and elevating the harvest indices of crop cultivars. Although there have been numerous achievements in the development of dwarfism and plant type in Brassica napus breeding, exploring new materials conferring oilseed rape with efficient plant types that provide higher yields is still of significance in breeding, as well as in elucidating the mechanisms underlying plant development. Here, we report a new dwarf architecture with down-curved leaf mutant (Bndwf/dc/1) isolated from an ethyl methanesulphonate (EMS)-mutagenized B. napus line, together with its inheritance and gene mapping, and pleiotropic effects of the mapped locus on plant-type traits.

Results: We constructed a high-density single-nucleotide polymorphism (SNP) map using a backcross population derived from the Bndwf/dcl1 mutant and the canola cultivar 'zhongshuang11' ('ZS11') and mapped the dwarf architecture with the down-curved leaf dominant locus, BnDWF/DCL1, in a 6.58-cM interval between SNP marker bins M46180 and M49962 on the linkage group (LG) C05 of B. napus. Further mapping with other materials derived from Bndwf/dcl1 narrowed the interval harbouring BnDWF/DCL1 to $175 \mathrm{~kb}$ in length and this interval contained 16 annotated genes. Quantitative trait locus (QTL) mappings with the backcross population for plant type traits, including plant height, branching height, main raceme length and average branching interval, indicated that the mapped QTLS for plant type traits were located at the same position as the BnDWF/DCL1 locus.
\end{abstract}

Conclusions: This study suggests that the BnDWF/DCL1 locus is a major pleiotropic locus/QTL in B. napus, which may reduce plant height, alter plant type traits and change leaf shape, and thus may lead to compact plant architecture. Accordingly, this locus may have substantial breeding potential for increasing planting density.

Keywords: Brassica napus, Dwarf architecture with down-curved leaf mutant, Single-nucleotide polymorphism, Gene mapping

Abbreviations: 'ZS11', 'Zhongshuang11'; Bndwf/dcl1, Dwarf architecture with down-curved leaf mutant; BR, Brassinosteroid; CTAB, Cetyl trimethylammonium bromide; EMS, Ethyl methanesulfonate; GA, Gibberellic acid; ICIM, Inclusive composite interval mapping; LG, Linkage groups; LOD, Logarithm of odds; PCR, Polymerase chain reaction; QTL, Quantitative trait locus; SNP, Single-nucleotide polymorphism; SSR, Simple sequence repeat

\footnotetext{
* Correspondence: guanrzh@njau.edu.cn

${ }^{\dagger}$ Equal contributors

${ }^{1}$ State Key Laboratory of Crop Genetics and Germplasm Enhancement, Nanjing Agricultural University, Nanjing 210095, China

${ }^{2}$ Jiangsu Collaborative Innovation Center for Modern Crop Production, Nanjing, Jiangsu, China
} 


\section{Background}

Traits related to plant height or compact plant type are very important due to their role in enhancing lodging resistance or the planting density in crops [1-4]. Certain key genes related to plant type traits have played very important roles in crop genetic improvement. In wheat, the Rht (Reduced height) genes controlling a key step in the signal transduction pathway of the growth hormone gibberellic acid (GA), have been utilized worldwide, bringing about the "green revolution" in crop production [1]. In rice, a semi-dwarf gene $s d 1$ that regulates a key step in the biosynthesis of GA [5-7] has proved extremely important in elevating harvest index and lodging resistance in worldwide rice production. However, the mechanism underlying the development of plant type or dwarfism is complex because many loci related to plant hormone biosynthesis and signal transduction [e.g., GA, brassinosteroid (BR) and auxin] and transcription factors, might determine plant height and architecture [8-15].

Rapeseed is one of the crops prone to lodging, which can lead to yield loss and difficulty in harvesting, and thus scientists have paid considerable attention to dwarfism in B. napus [16-19]. The dwarf gene $B z h$, which was derived from the cultivar 'Primor' through chemical mutagenesis, was first identified and later mapped in 1995 $[16,17]$. The dwarf gene $B z h$ has an additive effect, which may bring about a greater than $30 \%$ reduction in plant height $[16,17]$. Using this gene, several dwarf or semi-dwarf rapeseed cultivars, such as 'Bienvenu-bzh', '2405-bzh' and 'Darmor-bzh' (with formally released genome sequences), have been raised. Muangprom et al. [20-22] identified and studied the dwarf gene Brrga1-d on the A06 chromosome of Brassica rapa, which encodes a DELLA protein, and transferred this to B. napus cultivars. Experiments have demonstrated that this gene may reduce plant height and elevate lodging resistance due to an altered GA signaling pathway. Later, the semidwarf gene $D S-1$ in B. napus was mapped to chromosome A06. Molecular experiments have demonstrated that $D S-1$ encodes a DELLA protein in which a single proline $(\mathrm{P})$ to leucine $(\mathrm{L})$ substitution in the VHYNP motif leads to dwarf mutation, a gain-of-function mutation in GA signaling [18]. In another study, the dwarfism of $B$. napus banC.dwf, was found to be controlled by one recessive gene that leads to insensitivity to exogenous GA3 [19]. In addition to the aforementioned dwarfism related to GA biosynthesis or signaling, dwarfism related BR signaling or other pathways has also been identified. The phenotype of the B. napus dwarf mutant 'NDF-1' was found to be controlled by a major gene possessing a mainly additive effect and a non-significant dominance effect, and a three-base mutation in the pyrimidine box (P-box) of the BnGID1promoter was found to be linked to its dwarf phenotype $[23,24]$. Recently, the dominant
BnDWF1 locus on chromosome BnA09 has been found to be associated with the B. napus dwarf mutant, Bndwf1 [25].

Although there have been numerous achievements in the exploration of dwarfism and plant type breeding in $B$. napus, exploring new materials that confer oilseed rape with efficient plant types leading to considerably higher yields is still of significance in breeding, as well as in elucidating the mechanisms underlying plant development. New materials with specific plant type traits, such as compact plant architecture and shorter plant height, are worthy of investigation. The present study describes a dominant dwarf architecture with down-curved leaf mutant (Bndwf/dcl1) isolated from an EMS-mutagenized $B$. napus line, together with its inheritance, gene mapping and effects on the agronomic traits. Additionally, to map the dominant BnDWF/DCL1 locus and QTLs for plant type-related traits, we constructed a saturated SNP linkage map. Our findings may offer insight into elucidating the molecular mechanism underlying the dominant compact plant type and down-curved leaf phenotype and identification of the key gene controlling the plant height and down-curved leaf trait in B. napus.

\section{Results}

\section{Performance of the dwarf down-curved leaf mutant}

At the seedling stage, leaves of the Bndwf/dcl1 mutant have a sharply down-curved and crinkled phenotype, with short petioles, which contrasts with the wild-type leaves that are normal with long petioles (Fig. 1a and b). The leaves of the adult Bndwf/dcl1 mutant become slightly down-curved and crinkled before flowering, and are slightly down-curved but not crinkled after flowering. At the mature stage, the Bndwf/dcl1 mutant plant exhibits a compact dwarf plant type, while the wild-type (Fig. 1c) exhibits a tall plant type. Plant height of the Bndwf/dcl1 mutant was only $40-70 \mathrm{~cm}$, which is considerably shorter than that of the wild-type (approximately $1.5 \mathrm{~m}$ ).

\section{Inheritance of the dwarf architecture with down-curved leaf trait}

The $\mathrm{F}_{1}$ plants of 'ZS11' $\times$ Bndwf/dcl1 had down-curved leaves and dwarf plant height, indicating that the dwarfism and down-curved leaves are dominant traits. The plants in the $\mathrm{BC}_{1}$ population could be divided into two different groups: dwarf plants with down-curved leaves, and tall plants with normal flat leaves (Fig. 1). The 423 $\mathrm{BC}_{1}$ plants contained 223 dwarf plants with downcurved leaves and 200 normal plants with normal leaves. A chi-squared test indicated that the segregation pattern agreed with the Mendelian segregation ratio of 1:1 (Table 1). In the $F_{2}$ population, the segregation obeyed the Mendelian segregation ratio of 1:3 (normal plants with normal flat leaves: dwarf plants with down-curved leaves) (Table 1$)$. The reciprocal $F_{2}$ population $\left(R F_{2}\right)$ was 

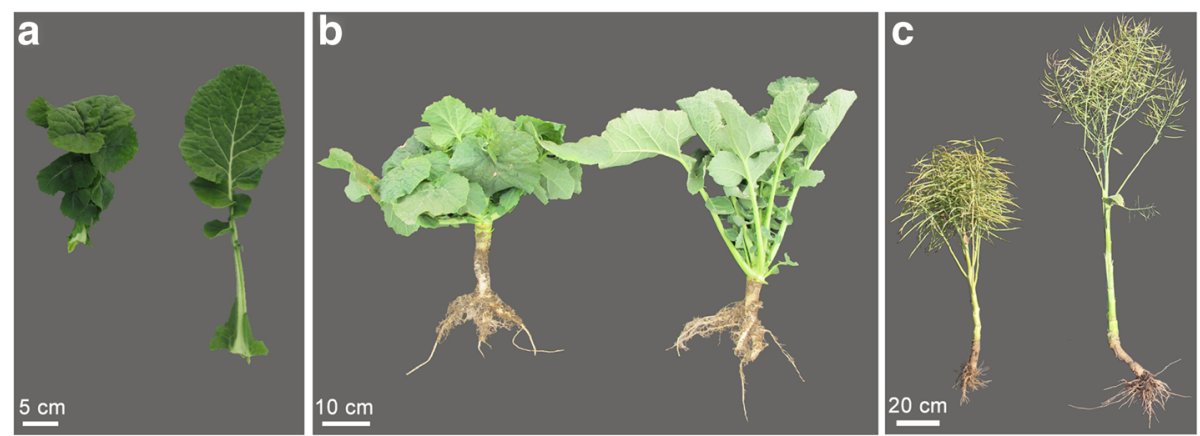

Fig. 1 Morphological charactersof the Bndwf/dcl1 mutant and its wild-type. a Leaf phenotype at the seedling stage of the Bndwf/dcl1 mutant (left) and its wild-type (right). $\mathbf{b}$ Phenotype at the seedling stage of the Bndwf/dcll mutant (left) and its wild-type (right). c Phenotype at the mature stage of the Bndwf/dcl1 mutant (left) and its wild-type (right)

verified to have the same inheritance segregation mode as the $\mathrm{F}_{2}$ population (Table 1). Thus, we infer that the dwarf architecture with down-curved leaf trait is controlled by a single dominant gene.

Several plant type-related traits were investigated in the backcross population. It was found that the plants with down-curved leaves were consistently dwarf and compact in plant type. For plants with down-curved leaves, measurements for plant height, branching height, main raceme length and average branching interval, were all significantly smaller than those of the plants with normal leaves (Table 2). The plant height was on average reduced by approximately $60 \%$ compared with the normal plants. Plant height was significantly correlated with branching height, main raceme length and average branching interval, with correlation coefficients $r=0.868^{* * *}, 0.945^{* * *}$ and $0.736^{* * *}$ respectively (** denotes significant at the 0.01 level, see Additional file 1). These results clearly indicate that the down-curved leaf trait is accompanied by compact plant type architecture by reduced branching height, main raceme length and branching interval. However, the compact type of plant architecture does not necessarily indicate reduction in the number of the primary branches (Table 2). Additionally, in the $\mathrm{BC}_{1}$ population, the 1000 -seed weight and yield per plant of dwarf plants with down-curved leaves were both significantly less than those of the normal plants with normal leaves (Table 2).

\section{Construction of a high density SNP map}

With the $\mathrm{BC}_{1}$ population derived from 'ZS11' $\times($ 'ZS11' $\times$ Bndwf/dcl1), we genotyped 109 plants. Although the
Brassica $60 \mathrm{~K}$ SNP BeadChip has 52,157 SNP markers, after deleting invalid markers, only 14,682 polymorphic markers were used to construct the linkage map. Nineteen LGs obtained by JoinMap 4 software contained 818 bins representing 7489 markers (for detailed data see Additional file 2). The total length of the map was $1583.05 \mathrm{cM}$, the longest LG was C03 at $134.54 \mathrm{cM}$ and the shortest LG was C09 at only $22.91 \mathrm{cM}$ (Table 3). The mean interval between adjacent markers was $1.98 \mathrm{cM}$. This high-density map may be used for mapping the BnDWF/DCL1 locus and the loci of other agronomic traits.

\section{Mapping of BnDWF/DCL1}

After building the saturated SNP genetic map, the $B n D W F / D C L 1$ locus was mapped onto LG C05, positioned in an interval of $6.58 \mathrm{cM}$ between SNP bins M46180 and M49962 (Fig. 2a). A search of the B. napus genome database had shown that SNP probe sequences of the two flanking markers matched completely with their physical position on chromosome $\mathrm{C} 05$ in B. napus cv. 'Darmor- $b z h$ '. On the basis of the genome sequence of B. napus cv. 'Darmor-bzh', the BnDWF/DCL1 locus is inferred to reside within a region of $16.354 \mathrm{Mb}$ on $\mathrm{C} 05$ of B. napus cv. 'Darmor-bzh'. LG C05 was 64.75 cM long and contained 26 bins representing 187 SNP markers, indicating that the chromosome C05 of B. napus had enriched SNP markers in our population. Unfortunately, the target interval harbouring the BnDWF/DCL1 locus did not have polymorphic SNP markers, leading to a failure in identifying the nearest recombination site by using this population.

Table 1 Inheritance of the dwarf architecture with down-curved leaf trait of Bndwf/dcl1

\begin{tabular}{|c|c|c|c|c|c|}
\hline Population & No. of normal plants with normal leaves & No. of dwarf plants with down-curved leaves & Expected segregation & $x^{2}$ & $p$ value \\
\hline $\mathrm{BC}_{1}$ & 200 & 223 & $1: 1$ & 1.14 & 0.29 \\
\hline $\mathrm{F}_{2}$ & 126 & 319 & $1: 3$ & 2.43 & 0.12 \\
\hline $\mathrm{RF}_{2}$ & 97 & 247 & $1: 3$ & 1.71 & 0.19 \\
\hline
\end{tabular}


Table 2 Agronomic performance of the Brassica napus 'ZS11' $\times\left(\right.$ 'ZS11' $\times$ Bndwf/dcl1) $\mathrm{BC}_{1}$ population

\begin{tabular}{|c|c|c|c|c|c|c|}
\hline \multirow[t]{2}{*}{ Trait } & \multirow[t]{2}{*}{ 'ZS11' } & \multirow[t]{2}{*}{ Bndwf/dcl1 } & \multirow[t]{2}{*}{$\mathrm{F}_{1}$} & \multicolumn{3}{|l|}{$\mathrm{BC}_{1}$ population } \\
\hline & & & & $\begin{array}{l}\text { Normal plants with normal } \\
\text { leaves }\end{array}$ & $\begin{array}{l}\text { dwarf plants with down-curved } \\
\text { leaves }\end{array}$ & $p$ value \\
\hline Plant height & $143.5 \pm 7.0$ & $49.3 \pm 8.7$ & $56.2 \pm 2.6$ & $122.5 \pm 13.0$ & $48.2 \pm 8.0^{b}$ & 1.7E-237 \\
\hline Branching height & $29.2 \pm 3.5$ & $9.9 \pm 3.8$ & $11.9 \pm 3.8$ & $34.0 \pm 7.6$ & $10.2 \pm 3.3^{b}$ & $2.2 \mathrm{E}-155$ \\
\hline Main raceme length & $72.3 \pm 6.6$ & $24.4 \pm 4.2$ & $29.6 \pm 5.1$ & $57.9 \pm 9.3$ & $25.3 \pm 6.1^{b}$ & $1.6 \mathrm{E}-156$ \\
\hline $\begin{array}{l}\text { Average branching } \\
\text { interval }\end{array}$ & $6.6 \pm 1.0$ & $3.4 \pm 2.1$ & $3.5 \pm 1.4$ & $7.1 \pm 2.0$ & $3.2 \pm 1.6^{b}$ & $5.5 \mathrm{E}-76$ \\
\hline No. of primary branches & $7.4 \pm 1.2$ & $5.7 \pm 1.1$ & $5.3 \pm 0.9$ & $5.4 \pm 1.4$ & $5.2 \pm 1.4$ & 0.22 \\
\hline Yield per plant ${ }^{a}$ & $24.3 \pm 4.1$ & $14.0 \pm 2.9$ & $14.9 \pm 3.1$ & $18.5 \pm 11.2$ & $14.7 \pm 8.4^{b}$ & 0.00011 \\
\hline 1000-seed weight ${ }^{a}$ & $4.570 \pm 0.304$ & $3.426 \pm 0.702$ & $4.221 \pm 0.394$ & $4.364 \pm 0.521$ & $3.924 \pm 0.660^{b}$ & $3.28 \mathrm{E}-13$ \\
\hline
\end{tabular}

${ }^{a}$ Indicates that the trait with this sign was investigated in 20 individuals randomly sampled from dwarf and tall subpopulations, respectively

${ }^{b}$ Indicates significant differences between dwarf and tall plants at the 0.01 level by $t$-test. Data are presented as mean \pm standard deviation (SD)

\section{QTL mapping related to plant type}

In addition to mapping of $B n D W F / D C L 1$, we also mapped QTLs for agronomic traits in the $\mathrm{BC}_{1}$ population. As a result, we identified a single major QTL for plant height on LG C05, termed qPHC05, which explained $79.5 \%$ of the phenotypic variation in plant height (Table 4). It apparently mapped at the same position as the BnDWF/DCL1 locus, had a large LOD score of 35.0 (see Additional file 3), and had a high genetic effect $(-62.30 \mathrm{~cm})$. This probably indicates the BnDWF/ DCL1 locus (QTL) not only causes changes in leaf shape, but also reduces plant height in plants carrying the dominant BnDWF/DCL1 locus. Furthermore, we identified a QTL for branching height ( $q B H C 05)$, a QTL for main raceme length ( $q M R L C 05$ ), and a QTL for average branching interval (qABIC05). These QTLs of traits related to plant type were at approximately the same position as BnDWF/DCL1. They consistently had high LOD values (see Additional file 3), obvious genetic effects and high phenotypic variation explained (PVE) percentages (Table 4). Thus, we conclude that the BnDWF/ DCL1 locus (QTL) probably has a pleiotropic effect on

Table 3 Statistics of the LGs constructed from the $\mathrm{BC}_{1}$ population of Brassica napus

\begin{tabular}{|c|c|c|c|c|c|c|}
\hline LG & Bin & Marker & Length (cM) & Mean interval (cM) & Max interval (cM) & Min interval (cM) \\
\hline $\mathrm{A} 01$ & 51 & 403 & 75.67 & 1.51 & 9.67 & 0.11 \\
\hline $\mathrm{A} 02$ & 34 & 298 & 110.88 & 3.36 & 18.44 & 0.66 \\
\hline A03 & 66 & 439 & 133.96 & 2.06 & 16.06 & 0.27 \\
\hline $\mathrm{A} 04$ & 46 & 591 & 92.24 & 2.05 & 19.74 & 0.17 \\
\hline A05 & 66 & 630 & 95.63 & 1.47 & 12.14 & 0.10 \\
\hline A06 & 62 & 342 & 122.12 & 2.00 & 14.69 & 0.19 \\
\hline A07 & 48 & 323 & 71.47 & 1.52 & 7.85 & 0.17 \\
\hline $\mathrm{A} 08$ & 34 & 416 & 41.08 & 1.24 & 4.34 & 0.08 \\
\hline A09 & 66 & 599 & 119.57 & 1.84 & 11.19 & 0.21 \\
\hline A10 & 24 & 129 & 47.24 & 2.05 & 12.79 & 0.24 \\
\hline $\mathrm{C} 01$ & 33 & 447 & 86.53 & 2.70 & 19.09 & 0.23 \\
\hline C02 & 60 & 622 & 71.36 & 1.21 & 10.68 & 0.14 \\
\hline $\mathrm{CO3}$ & 61 & 628 & 134.54 & 2.24 & 18.00 & 0.38 \\
\hline CO4 & 33 & 489 & 58.66 & 1.83 & 6.85 & 0.20 \\
\hline C05 & 26 & 187 & 64.75 & 2.59 & 13.37 & 0.44 \\
\hline C06 & 27 & 289 & 57.19 & 2.20 & 10.25 & 0.26 \\
\hline C07 & 34 & 242 & 65.09 & 1.97 & 10.50 & 0.36 \\
\hline C08 & 36 & 303 & 112.16 & 3.20 & 15.38 & 0.24 \\
\hline CO9 & 11 & 113 & 22.91 & 2.29 & 9.48 & 0.68 \\
\hline Total & 818 & 7489 & 1583.05 & & & \\
\hline
\end{tabular}




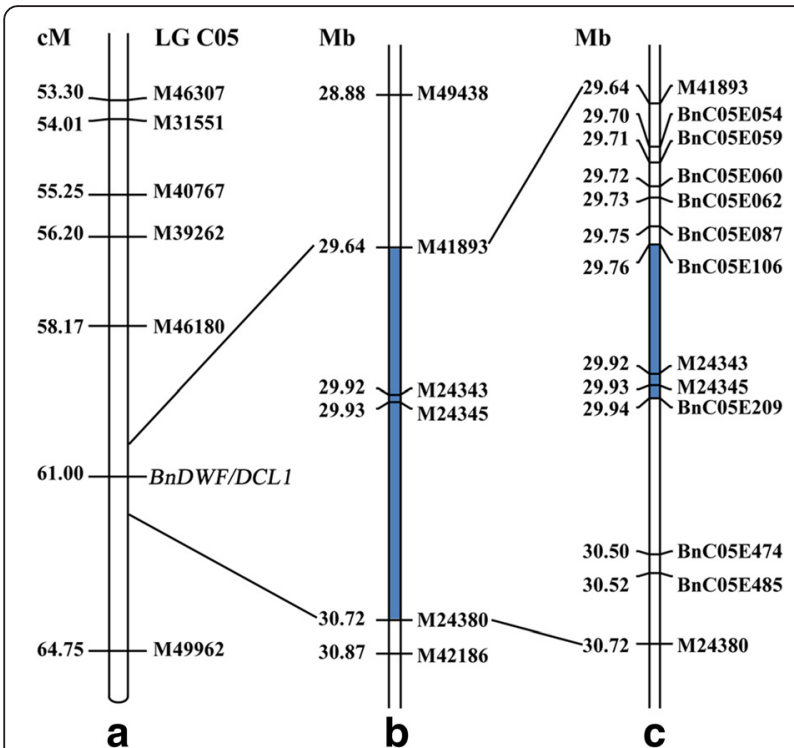

Fig. 2 Mapping of BnDWF/DCL1 with SNP and SSR markers using $\mathrm{BC}_{1}$ and $\mathrm{F}_{5}$ populations. a The BnDWF/DCL1 locus was mapped by SNP marker at position of $61.00 \mathrm{cM}$ of $\mathrm{LG}$ C05 in the $\mathrm{BC}_{1}$ population, which was in a 6.58-cM region between M46180 and M49962. b SNP screening of $29 \mathrm{~F}_{5}$ individuals narrowed the BnDWF/DCL 1 locus to the interval between M41893 and M24830 (1.08 Mb, the blue segment) of chromosome C05 in B. napus cv. 'Darmor-bzh', and this was co-segregated with M24343 and M24345. c SSR marker screening resulted in further mapping of the BnDWF/DCL 1 locus to the interval between SSR markers BnC05E106 and BnC05E209 (175 kb)

plant type. In other words, the dominant locus $B n D W F /$ $D C L 1$ causes elemental shrinkages in the plant architecture, leading to pleiotropic effects on different parts of plants. It is noted that the position of this dwarf locus together with its related traits are all different from the reported dwarf QTLs in B. napus.

\section{Further mapping of the BnDWF/DCL1 locus}

The above-mentioned mapping interval for BnDWF/ $D C L 1$ was too long to be of value for further studies due to the lack of polymorphic SNP markers in the mapping interval. To solve this problem, we investigated $29 \mathrm{~F}_{5}$ individuals derived from the cross between the European rapeseed cultivar 'Tapidor' and Bndwf/dcll. Genotyping the $29 \mathrm{~F}_{5}$ individuals using the Brassica $60 \mathrm{~K} \mathrm{SNP}$ BeadChip Array revealed that the $B n D W F / D C L 1$ locus co-segregated with SNP markers M24343 and M24345, and seven recombinants were found among the $29 \mathrm{~F}_{5}$ individuals. Of these, six recombinants with down-curved leaves were observed be recombined between M24343 and M41893, and one line with down-curved leaves was observed to show recombination between M24345 and M24380. Information from markers M41893, M24343, M24345 and M24380 helped narrow the mapping interval to a $1.08-\mathrm{Mb}$ region between M41893 and M24380, and there were no other polymorphic SNP markers in this region (Fig. 2b).

On the basis of this revised mapping interval and its corresponding genome sequence, 155 simple sequence repeat (SSR) markers were designed to clarify the recombination site by polymerase chain reaction (PCR). Nine of the designed SSR markers were found to be polymorphic: BnC05E054, BnC05E059, BnC05E060, BnC05E062, BnC05E087, BnC05E106, BnC05E209, BnC05E474 and BnC05E485 (see Additional file 4). By genotyping the seven $\mathrm{F}_{5}$ recombinant individuals using these nine polymorphic markers, fortunately, we found that the BnDWF/DCL1 locus can be mapped to the interval between BnC05E106 and BnC05E209, based on recombination site analysis with SSR markers. The relative orders and positions of all the polymorphic SNP and SSR markers on LG C05 and chromosome C05 of $B$. napus cv 'Darmor-bzh' are unanimous (Fig. 2). Accordingly, the mapping interval between BnC05E106 and BnC05E209 was identified to be $175 \mathrm{~kb}$ in length on basis of their positions on the C05 chromosome of $B$. napus cv. 'Darmor-bzh' (Fig. 2c). No polymorphic SSR marker other than the nine SSR markers can be found in this small region, which was progressively determined by our careful investigation.

To validate these results, the remaining plants of the $F_{5}$ family populations were genotyped using the SSR markers. PCR detection in the segregating or homologous $F_{5}$ family populations produced results that were consistent with those of previous experiments, including phenotype observations and marker genotyping. Thus we may conclude that the $B n D W F / D C L 1$ locus is located in a 175-kb interval between BnC05E106 and BnC05E209 on chromosome C05 of B. napus cv. 'Darmor-bzh'.

Additionally, our analysis for the fine mapping of the $B n D W F / D C L 1$ locus was based on a formally released genome sequence database of $B$. napus cv. 'Darmor-bzh'. Nevertheless, segments homologous to that harbouring the $B n D W F / D C L 1$ locus cannot be found in the genome

Table 4 QTLs identified for agronomic traits in the BC 1 population derived from parents 'ZS11' and Bndwf/dcl1

\begin{tabular}{llllllcc}
\hline Trait & QTL & LG & Position & Marker interval & LOD & Effect & PVE (\%) \\
\hline Plant height & qPHC05 & C05 & 61.1 & M46180-M49962 & 35.0 & -62.30 & 79.5 \\
Branching height & qBHC05 & C05 & 61.0 & M46180-M49962 & 26.8 & -20.28 & 71.2 \\
Main raceme length & qMRLC05 & C05 & 61.0 & M46180-M49962 & 22.3 & -27.37 & 65.2 \\
Average branching interval & qABIC05 & C05 & 61.5 & M46180-M49962 & 10.9 & -3.82 & 41.4 \\
\hline
\end{tabular}


database of B. napus cv. 'ZS11'. However, in this database, we did find two highly homologous matches to the mapping segment, which were a segment of $86.78 \mathrm{~kb}$ in length on chromosome C04 (from 25534021 to 25620800), and a segment of $91.6 \mathrm{~kb}$ in length on chromosome C05 (from 29448801 to 29540400 ) (Additional file 5). Theoretically, it is of very small probability to depart two segments homologous to an entire segment by an evolutionary event at recent time. Thus, it may be inferred that the disparity between two public sources of genome information regarding this region is due to an error that occurred in assembly of the genomes of the allotetraploid species B. napus. However, strong systematic evidence from the aforementioned SNP and SSR marker experiments, such as recombination percentage and marker cosegregation information, consistently support our contention that the results based on the genome database of $B$. napus $\mathrm{cv}$. 'Darmor-bzh' are reliable for this work. Analysis of the annotation information of the 175-kb mapping interval revealed that the chromosomal segments harbouring the $B n D W F / D C L 1$ locus in $B$. napus cv. 'Darmor-bzh' contain 16 annotated genes found in public databases (http://brassicadb.org/brad/ downloadOverview.php) (Additional file 6).

\section{Discussion}

Although studies of plant type regulation in plant species have been intensified, the new applicable gene is rare. Mining the key genes related to plant type is still of significance in many crops. Compact plant type may be used to increase planting density in B. napus, with the aim of elevating yield per unit area. A compact plant type together with the down-curved leaves phenotype was observed in a population derived from the Bndwf/ dcl1 mutant. The BnDWF/DCL1 locus position and its related traits are all different from that of the reported dwarf QTLs in B. napus [16-25]. The BnDWF/DCL1 locus has a more obvious effect on reducing plant height than these reported rapeseed dwarf loci and confers plants with compact architecture, thus, this will certainly be of value in breeding a variety with compact plant type because the moderate penalty of the dwarfism may possibly be compensated or overcome by increased planting density. However, in the future, the breeding potential of the Bndwf/dcl1 mutant will need to be explored with attention paid to breeding strategies, although we still consider that it will be of significance in oilseed rape breeding.

Use of SNP markers has been beneficial to plant genotyping efforts because of the numerous distinct markers and high genome coverage [26-28]. In B. napus, the Brassica $60 \mathrm{~K}$ SNP BeadChip Array has recently helped advance rapeseed research efforts, and enabled the efficient construction of several high-quality saturated linkage maps over a short period [25, 29-32]. By using this
SNP chip in the present study, we constructed a saturated $B$. napus map, with 818 bins containing 7489 markers and a total length of $1583.05 \mathrm{cM}$. The BnDWF/ $D C L 1$ locus was primarily mapped to a 6.58 -cM interval between M46180 and M49962 of B. napus chromosome C05. The SNP marker distance of $6.58 \mathrm{cM}$ corresponds to the physical map length (16.354-Mb). This indicated that the mapping interval may contain a centromere since the physical distance is not consistent with the observed phenomena that $1 \mathrm{cM}$ is on average equivalent to a genomic sequence length of $0.4-0.5 \mathrm{Mb}$ in $B$. napus.

Although our investigations were initially limited by the lack of polymorphic molecular markers in the target mapping interval, we fortunately had alternative accessions that were derived from the cross between 'Tapidor' and $B n d w f / d c l 1$, which have undergone recombination in the selfing and breeding process. The enriched materials prepared in this research helped us to progressively approach the target genes.

Compared with many other traditional mapping techniques, our mapping procedure included the additional step of homologous segment analysis. Indeed, this analysis is probably a necessary step in the mapping. Newly constructed public Brassica genome databases cannot entirely exclude the possibly of certain genome assembly errors, which may lead to the inaccurate mapping intervals. Analysis integrated with marker experimental information may help to perfect the mapping results, as this will clarify which sequences are more reliable in cases where there is disparity between two or more homologous genome sequence segments.

Various mechanisms underlying plant dwarfism have been reported. Genes participating in biosynthesis and signal transduction of plant hormones, such as GA, BR and auxin, have been related to dwarf plant architecture. Dwarfism may also be affected by homeotic genes and genes related to the cell wall, polyamine biosynthesis and transcription factors. The present study has revealed the genes within a designated mapping interval, which contains genes with functions similar to those reported to be associated with plant dwarfism. However, further studies are required to identify the gene responsible for the dwarf architecture with down-curved leaf mutant trait in B. napus.

\section{Conclusions}

The BnDWF/DCL1 locus was demonstrated to be associated with the dwarf architecture with down-curved leaf trait in B. napus. Construction of a high-density SNP map enabled us to position the BnDWF/DCL1 locus in a 6.58-cM interval on LG C05 of B. napus. Further mapping with other materials derived from Bndwf/dcl1 enabled us to narrow the interval for $B n D W F / D C L 1$ to 175-kb. QTL mapping indicated that BnDWF/DCL1 to 
be a major dominant locus that has pleiotropic effects on leaf type and changes in plant type traits. Our findings have revealed a key locus with plant type breeding potential, and may offer insights into elucidating the molecular mechanism underlying the dominant plant type in B. napus.

\section{Methods}

\section{Plant materials}

The B. napus dwarf architecture with down-curved leaf mutant, Bndwf/dcl1, was originally isolated from an EMS-mutagenized B. napus pure line, NJ7982, at Nanjing Agricultural University, China. The dwarf architecture with down-curved leaf mutant was crossed with canola variety 'ZS11', and then backcrossed with 'ZS11' to produce the $\mathrm{BC}_{1}$ generation. We phenotyped the $\mathrm{BC}_{1}$ population plants at the seedling and mature stages. Fourteen normal plant DNA samples, 95 down-curved leaf plant DNA samples, and DNA from the recessive recurrent parent, 'ZS11', were then used for SNP genotyping. Two reciprocal $F_{2}$ populations derived from the same cross were also used for genetic analysis.

One homologous selfing $F_{5}$ family (contains 153 dwarf plants with down-curved leaves) and three segregating selfing $F_{5}$ families (contain 14, 12 and 13 dwarf plants with down-curved leaves and 14, 8 and 5 normal plants with normal leaves, respectively) derived from the cross of "Tapidor $\times B n d w f / d c l 1$ " were used to further map the dwarf architecture with down-curved leaf locus. Some of plants with dwarf architecture in the segregating families died because they were shadowed by taller plants, thus the data of the segregating families were not used for chi-square test. Twenty-nine individuals ( 23 dwarf plants with down-curved leaves and 6 normal plants with normal leaves) from the four $F_{5}$ families were used for SNP marker genotyping with aim of further mapping the $B n D W F / D C L 1$ locus. All plants of these populations were used to identify recombination sites resulting from the breeding process, using SSR markers.

All materials were grown at the same density in fields of the Jiangpu Experimental Station at the Nanjing Agricultural University. Plants were sown uniformly in rows of $2.5 \mathrm{~m}$ length with 15 individuals in each row and $0.4 \mathrm{~m}$ spacing between rows. The $\mathrm{BC}_{1}$ and two reciprocal $F_{2}$ populations were grown in 2012 . The $F_{5}$ lines were grown in 2014.

\section{Agronomic traits observation}

In the $\mathrm{BC}_{1}$ population, agronomic traits, including plant height, branching height, main raceme length, number of first branches and average branching interval, were investigated in all the individuals, and 20 individuals randomly sampled from dwarf and tall subpopulations, respectively, were used to investigate the yield per plant and 1000-seed weight. Every eight individuals were randomly selected from the parents and $F_{1}$ of the $B_{1}$ population to examine all the agronomic traits.

The plant height $(P H)$ was measured from the ground to the top of the individual, the branching height $(B H)$ was measured from the ground to the first node, and the main raceme length $(M R L)$ was measured from the last branch base to the top of the plant. The average branching interval $(A B I)$ was calculated using the following formula:

$$
A B I=(P H-B H-M R L) /(\text { number of first branches }-1) .
$$

\section{Construction of a SNP genetic map}

Total DNA was extracted from fresh leaves using a modified cetyl trimethylammonium bromide (CTAB) method [33]. The DNA samples were diluted to $200 \mathrm{ng}$ $\mathrm{uL}^{-1}$ and then genotyped using the Brassica $60 \mathrm{~K} \mathrm{SNP}$ BeadChip Array. There are a total of 52,157 SNP markers in the Brassica $60 \mathrm{~K}$ SNP BeadChip Array, which is sufficient to genotype our DNA samples. DNA sample preparation, hybridization to the BeadChip and imaging of the arrays were performed by the Beijing Emei Tongde Development Co. Ltd (Beijing, China). Allele calling for each locus was performed using GenomeStudio genotyping software v2011.1 (Illumina, Inc.). Cluster definitions were based on genotype data from rapeseed individuals. The SNP markers were named using $\mathrm{M}$ plus index numbers assigned by GenomeStudio, which are presented in the text and their original names are listed in Additional file 2.

The polymorphic SNP markers were first sorted into different bins. The first marker within each bin was selected as the representative of the bin and was used to construct the linkage map with JoinMap 4 software [34]. The SNP markers were first grouped to different LGs at a recombination frequency of 0.22 using the "Population" function. The marker order and distances in each LG were calculated using the Regression mapping algorithm. The Kosambi function was used for calculating the cM map distances with a logarithm of odds (LOD) threshold of 1.0 and recombination frequency of 0.4 .

\section{Mapping of the BnDWF/DCL1 locus and QTLs for agronomic traits}

A genetic map was constructed from the backcross population using the Brassica $60 \mathrm{~K}$ SNP BeadChip Array data. Detection of the BnDWF/DCL1 locus and QTLs for plant type-related agronomic traits was performed for the $\mathrm{BC}_{1}$ population using the inclusive composite interval mapping (ICIM) method [35]. The LOD threshold for QTL detection was determined by permutation test analyses (1000 permutations, $5 \%$ overall error level). 
Further mapping of the BnDWF/DCL1 locus

On the basis of the constructed SNP map, the physical regions containing the $B n D W F / D C L 1$ locus were identified by aligning SNP probe sequences with the genomes of B. napus cv. 'Darmor-bzh' using BLASTN (http:// blast.ncbi.nlm.nih.gov/). The locus of the B. napus cv. 'Darmor- $b z h$ ' genome, where the genome sequence has $100 \%$ identity with the SNP probe sequence, is the perfect position of the corresponding SNP marker on it. The Brassica $60 \mathrm{~K}$ SNP BeadChip Array was used to genotype the $29 \mathrm{~F}_{5}$ individuals derived from the cross between 'Tapidor' and Bndwf/dcl1.

Following gene mapping by these methods, the interval sequences of approximately $1 \mathrm{Mb}$ covering the significant SNP markers related to the mutant locus were downloaded from http://www.genoscope.cns.fr/brassicanapus/data/ for bioinformatics analysis. On the basis of the sequences of $B$. napus cv. 'Darmor-bzh' physical region containing the BnDWF/DCL1 locus, 637 SSR loci were identified using SSRHunter 1.3 software [36] with a 6-bp motif maximum and three-repeat minimum. Of these, 155 SSR loci with a 150-bp sequence on both sides were selected to design primers using the Primer Premier 5.0 software [37] (see Additional file 7). The PCR conditions were as follows: denaturation at $95{ }^{\circ} \mathrm{C}$ for $10 \mathrm{~min}$, followed by 35 cycles of $95^{\circ} \mathrm{C}$ for $30 \mathrm{~s}$, annealing for $40 \mathrm{~s}$ (the annealing temperature of each SSR marker is listed in Additional file 7), and $72{ }^{\circ} \mathrm{C}$ for $40 \mathrm{~s}$, and a final extension step at $72{ }^{\circ} \mathrm{C}$ for $10 \mathrm{~min}$.

The nine polymorphic markers identified from these 155 designed SSR markers were used for genotyping the $\mathrm{F}_{5}$ lines derived from a crosses between 'Tapidor' and the Bndwf/dcl1 mutant, to determine the recombination site in the breeding materials with the aim of narrowing the mapping interval.

\section{Analysis of genes in the mapping interval of the BnDWF/ $D C L 1$ locus}

Whole genome sequences of B. napus cv. 'Darmor-bzh' were downloaded from public databases (http://www.genoscope.cns.fr/brassicanapus/) [38]. On the basis of the positions of SSR and SNP markers on the genome, the corresponding physical region of mapping interval could be obtained. A bioinformatics analysis of annotated genes in the mapping region was then completed.

\section{Additional files}

Additional file 1: Table S1. Full list of the values of agronomic traits in the $\mathrm{BC}_{1}$ population. (XLSX $58 \mathrm{~kb}$ )

Additional file 2: Table S2. Detailed SNP genetic map. (XLSX 296 kb)

Additional file 3: Figure S1. Major QTLs for plant-type related traits mapped on LG C05 of the Brassica napus. (DOCX 89 kb)
Additional file 4: Figure S2. Polymorphism identification of the SSR markers. (DOCX $1028 \mathrm{~kb}$ )

Additional file 5: Figure S3. Dot matrix of the BnDWF/DCL1 mapping interval of B. napus cv. 'Darmor-bzh' to B. napus cv. 'ZS11'. (DOCX $284 \mathrm{~kb}$ )

Additional file 6: Table S3. Genes on the mapped segments of chromosome C05 of Brassica napus. (XLSX $22 \mathrm{~kb}$ )

Additional file 7: Table S4. Information of designed SSR markers. (XLSX $25 \mathrm{~kb})$

\section{Acknowledgments}

We thank Prof. Shengyi Liu and Prof. Wei Hua in Oil Crops Research Institute of the Chinese Academy of Agricultural Sciences for their guidance and support in SNP markers analysis.

\section{Funding}

This research was supported financially by the National Natural Science Foundation of China (31301352 and 31270386), the Fundamental Research Funds for the Central Universities of China (KYZ201202-7 and KJQN201423), the Jiangsu Agricultural Science and Technology Innovation Fund (JASTIF) (CX (14)2003), and the Priority Academic Program Development of Jiangsu Higher Education Institutions (PAPD) of China.

\section{Availability of data and materials}

All data generated or analyzed during this study are included in this published article and its supplementary information files.

\section{Author's contributions}

YW conducted the map construction and locus mapping, drafted the MS; WC, SW, MY and MW co-finished the experiments; PC modified the MS; RG conceived and supervised the research and polished the MS. All authors have read and approved the manuscript.

\section{Competing interests}

The authors declared that they have no competing interests.

Consent for publication

Not applicable.

Ethics approval and consent to participate

This study required no ethics approval.

Received: 3 February 2016 Accepted: 5 August 2016

Published online: 18 August 2016

References

1. Peng J, Richards DE, Hartley NM, Murphy GP, Devos KM, Flintham JE, Beales J. Fish LJ, Worland AJ, Pelica F, et al. 'Green revolution' genes encode mutant gibberellin response modulators. Nature. 1999;400(6741):256-61.

2. Hedden P. The genes of the green revolution. Trends Genet. 2003;19(1):5-9.

3. Vanneste $S$, Friml J. Auxin: a trigger for change in plant development. Cell. 2009;136(6):1005-16

4. Clouse SD, Sasse JM. Brassinosteroids: essential regulators of plant growth and development. Annu Rev Plant Physiol Plant Mol Biol. 1998;49:427-51.

5. Monna L, Kitazawa N, Yoshino R, Suzuki J, Masuda H, Maehara Y, Tanji M, Sato M, Nasu S, Minobe Y. Positional cloning of rice semidwarfing gene, $s d-1$ : rice "green revolution gene" encodes a mutant enzyme involved in gibberellin synthesis. DNA Res. 2002;9(1):11-7.

6. Sasaki A, Ashikari M, Ueguchi-Tanaka M, Itoh H, Nishimura A, Swapan D, Ishiyama K, Saito T, Kobayashi M, Khush GS, et al. Green revolution: a mutant gibberellin-synthesis gene in rice. Nature. 2002;416(6882):701-2.

7. Spielmeyer W, Ellis MH, Chandler PM. Semidwarf ( $(s d-1)$, "green revolution" rice, contains a defective gibberellin 20-oxidase gene. Proc Natl Acad Sci. 2002;99(13):9043-8.

8. Sun TP, Kamiya Y. The Arabidopsis GA1 locus encodes the cyclase ent-kaurene synthetase A of gibberellin biosynthesis. Plant Cell. 1994;6(10):1509-18.

9. Winkler RG, Helentjaris T. The maize Dwarf3 gene encodes a cytochrome P450-mediated early step in gibberellin biosynthesis. Plant Cell. 1995;7(8): 1307-17. 
10. Helliwell CA, Chandler PM, Poole A, Dennis ES, Peacock WJ. The CYP88A cytochrome P450, ent-kaurenoic acid oxidase, catalyzes three steps of the gibberellin biosynthesis pathway. Proc Natl Acad Sci. 2001;98(4):2065-70.

11. Ross JJ, O'Neill DP, Wolbang CM, Symons GM, Reid JB. Auxin-gibberellin interactions and their role in plant growth. J Plant Growth Regul. 2001;20(4): 336-53.

12. Bishop GJ. Brassinosteroid mutants of crops. J Plant Growth Regul. 2003; 22(4):325-35.

13. Teale WD, Paponov IA, Palme K. Auxin in action: signalling, transport and the control of plant growth and development. Nat Rev Mol Cell Biol. 2006; 7(11):847-59.

14. Zhang C, Bai MY, Chong K. Brassinosteroid-mediated regulation of agronomic traits in rice. Plant Cell Rep. 2014;33(5):683-96.

15. Singh AP, Savaldi-Goldstein S. Growth control: brassinosteroid activity gets context. J Exp Bot. 2015:66(4):1123-32

16. Foisset $N$, Delourme $R$, Barret $P$, Renard M. Molecular tagging of the dwarf BREIZH (Bzh) gene in Brassica napus. Theor Appl Genet. 1995:91(5):756-61.

17. Barret P, Delourme R, Foisset N, Renard M. Development of a SCAR (sequence characterised amplified region) marker for molecular tagging of the dwarf BREIZH (Bzh) gene in Brassica napus L. Theor Appl Genet. 1998; 97(5-6):828-33.

18. Liu C, Wang J, Huang T, Wang F, Yuan F, Cheng X, Zhang Y, Shi S, Wu J, Liu K. A missense mutation in the VHYNP motif of a DELLA protein causes a semi-dwarf mutant phenotype in Brassica napus. Theor Appl Genet. 2010; 121(2):249-58.

19. Zeng X, Zhu L, Chen Y, Qi L, Pu Y, Wen J, Yi B, Shen J, Ma C, Tu J, et al. Identification, fine mapping and characterisation of a dwarf mutant (bnaC.dwf) in Brassica napus. Theor Appl Genet. 2011;122(2):421-8.

20. Muangprom A, Osborn TC. Characterization of a dwarf gene in Brassica rapa, including the identification of a candidate gene. Theor Appl Genet. 2004;108(7):1378-84

21. Muangprom A, Thomas SG, Sun TP, Osborn TC. A novel dwarfing mutation in a green revolution gene from Brassica rapa. Plant Physiol. 2005;137(3):931-8.

22. Muangprom A, Mauriera I, Osborn TC. Transfer of a dwarf gene from Brassica rapa to oilseed B. napus, effects on agronomic traits, and development of a 'perfect' marker for selection. Mol Breed. 2006;17(2):101-10.

23. Wang ML, Zhao Y, Chen F, Yin XC. Inheritance and potentials of a mutated dwarfing gene ndf1 in Brassica napus. Plant Breed. 2004;123(5):449-53.

24. Li H, Wang Y, Li X, Gao Y, Wang Z, Zhao Y, Wang M. A GA-insensitive dwarf mutant of Brassica napus L. correlated with mutation in pyrimidine box in the promoter of GID1. Mol Biol Rep. 2011;38(1):191-7.

25. Wang $Y$, He J, Yang L, Wang Y, Chen W, Wan S, Chu P, Guan R. Fine mapping of a major locus controlling plant height using a high-density single-nucleotide polymorphism map in Brassica napus. Theor Appl Genet 2016;129(8):1479-91.

26. Cho RJ, Mindrinos M, Richards DR, Sapolsky RJ, Anderson M, Drenkard E, Dewdney J, Reuber TL, Stammers M, Federspiel N, et al. Genome-wide mapping with biallelic markers in Arabidopsis thaliana. Nat Genet. 1999; 23(2):203-7.

27. Colasuonno P, Gadaleta A, Giancaspro A, Nigro D, Giove S, Incerti O, Mangini G, Signorile A, Simeone R, Blanco A. Development of a high-density SNP-based linkage map and detection of yellow pigment content QTLs in durum wheat. Mol Breed. 2014;34(4):1563-78.

28. Gaur R, Azam S, Jeena G, Khan AW, Choudhary S, Jain M, Yadav G, Tyagi AK Chattopadhyay D, Bhatia S. High-throughput SNP discovery and genotyping for constructing a saturated linkage map of chickpea (Cicer arietinum L.). DNA Res. 2012;19(5):357-73.

29. Fu Y, Wei D, Dong H, He Y, Cui Y, Mei J, Wan H, Li J, Snowdon R, Friedt W, et al. Comparative quantitative trait loci for silique length and seed weight in Brassica napus. Sci Rep. 2015;5:14407.

30. Liu J, Hua W, Hu Z, Yang H, Zhang L, Li R, Deng L, Sun X, Wang X, Wang H. Natural variation in ARF18 gene simultaneously affects seed weight and silique length in polyploid rapeseed. Proc Natl Acad Sci. 2015;112(37):5123-32.

31. Zhang D, Hua Y, Wang X, Zhao H, Shi L, Xu F. A high-density genetic map identifies a novel major QTL for boron efficiency in oilseed rape (Brassica napus L.). PLoS One. 2014;9(11):e112089.

32. Wang $Y$, He $Y$, Yang $M$, He J, Xu $P$, Shao M, Chu P, Guan R. Fine mapping of a dominant gene conferring chlorophyll-deficiency in Brassica napus. Sci Rep. 2016:6:31419.
33. Porebski S, Bailey LG, Baum BR. Modification of a CTAB DNA extraction protocol for plants containing high polysaccharide and polyphenol components. Plant Mol Biol Rep. 1997;15(1):8-15.

34. Ooijen JWV. JoinMap 4, Software for the calculation of genetic linkage maps in experimental populations. Wageningen: Kyazma B.V.; 2006.

35. Wang J, Li H, Zhang L, Meng. L. Users' manual of QTL IciMapping version 3. 2. The quantitative genetics group, institute of crop science, Chinese academy of agricultural sciences (CAAS), Beijing 100081, China, and genetic resources program, international maize and wheat improvement center (CIMMYT), Apdo. Postal 6-641, 06600 Mexico, D.F., Mexico. 2012.

36. Li Q, Wan JM. SSRHunter: development of a local searching software for SSR sites. Hereditas (Beijing). 2005;27(5):808-10.

37. Singh VK, Mangalam AK, Dwivedi S, Naik S. Primer premier: program for design of degenerate primers from a protein sequence. Biotechniques. 1998;24(2):318-9.

38. Chalhoub B, Denoeud F, Liu S, Parkin IA, Tang H, Wang X, Chiquet J, Belcram H, Tong C, Samans B, et al. Early allopolyploid evolution in the post-Neolithic Brassica napus oilseed genome. Science. 2014;345(6199):950-3.

\section{Submit your next manuscript to BioMed Central and we will help you at every step:}

- We accept pre-submission inquiries

- Our selector tool helps you to find the most relevant journal

- We provide round the clock customer support

- Convenient online submission

- Thorough peer review

- Inclusion in PubMed and all major indexing services

- Maximum visibility for your research

Submit your manuscript at www.biomedcentral.com/submit 